\title{
O USO DO SOFTWARE SCRATCH NO ENSINO DA GEOMETRIA
}

\section{THE USE OF SCRATCH SOFTWARE IN THE TEACHING OF GEOMETRY}

Carlos Henrique Damião dos Santos Filho

Universidade Federal de Mato Grosso Do Sul - UFMS.

E-mail: carloshenriquesantos00@outlook.com

RESUMO - Este trabalho apresenta o Software de programação Scratch como um método para contribuir com o ensino da Matemática. $O$ objetivo deste estudo foi realizar construções de ângulos, polígonos, cálculo de área e perímetro por meio de atividades práticas que serão orientadas pelo professor. A metodologia constou de estudo teórico, pesquisas bibliográficas, bem como o uso da tecnologia na educação. Além disso, foram feitas aplicações do estudo utilizando a ferramenta tecnológica. Procura apresentar as noções básicas de programações e realizar algumas atividades eficientes, de modo que os alunos possam interagir integralmente com a aula do professor que irá aplicar tal método de ensino. Com isso, espera-se que os estudantes adquiram os conhecimentos necessários que o software tem a oferecer, além de estimular a fixação do conteúdo e que o professor possa ministrar uma aula mais dinâmica, divertida e mais produtiva utilizando o recurso tecnológico que está à disposição para ser aproveitado na educação.

Palavras-chave: Software, Matemática, ensino.

ABSTRACT - In this paper present the Scratch programming software as a method to the teaching of Mathematics. The aim of this study was to carry out angles and polygons construction, area and perimeter calculations through practical activities that will be guided by the teacher. The methodology consisted of theoretical study, bibliographic research as well as the use of technology in education. In addition, the study's applications were made using the technological hardware. Demand to present the basics of programming and carry out some efficient activities, in such a way, that the students can fully interact with the class of the teacher who will apply such teaching method. With this, expected that students acquire the necessary knowledge that the software has to offer, in addition to stimulating the fixing of the content and the teacher can teach a more dynamic, fun and more productive class using the technological resource that is available to be used in education.

Keywords: Software, Mathematics, teaching. 


\section{INTRODUÇÃO}

Atualmente o uso da tecnologia em sala de aula não é mais algo surpreendente no mundo da educação, já que nossas crianças nascem em um mundo cada vez mais globalizado. Diante disso, sabe-se que a didática do professor deve andar lado a lado com os avanços tecnológicos, uma vez que, segundo o Centro Nacional de Estudos para o Desenvolvimento da Sociedade da Informação (CETIC), o mesmo mostrou que em 2017 97\% dos estudantes acessam a internet através de smartphones. Além disso, $81 \%$ das escolas públicas têm laboratórios de informática, mas apenas 59\% deles são usados, segundo Varella (2017).

No ano de 2017, a Câmara dos Deputados criou leis que favorecerem o uso da tecnologia nas instituições de ensino para que os professores possam ter mais um recurso no momento em que for lecionar. Essas leis têm como objetivo apoiar a universalização do acesso a internet, promover o uso de tecnologias digitais e proporcionar as condições necessárias para a implantação da tecnologia como instrumento pedagógico na educação básica.

Art. 1o Fica instituída a
Política de Inovação
Educação Conectada, em
consonância com a
estratégia 7.15 do Plano
Nacional de Educação,
aprovado pela Lei no 13.005,
de 25 de junho de 2014,
com o objetivo de apoiar a
universalização do acesso à
internet em alta velocidade
e fomentar o uso
pedagógico de tecnologias
digitais na educação básica.
Art. 20 A Política de
Inovação Educação
Conectada visa a conjugar
esforços entre órgãos e
entidades da União, dos
Estados, do Distrito Federal,
dos Municípios, escolas,
setor empresarial e
sociedade civil para
assegurar as condições
necessárias para a inserção
da tecnologia como
ferramenta pedagógica de

uso cotidiano nas escolas públicas de educação básica. (CÂMARA DOS DEPUTADOS, 2017.).

Por conta disso, considera-se importante que os professores façam uso dos recursos tecnológicos que estão disponíveis para seus alunos.

Os computadores e smartphones, assim como os tablets, possuem vários softwares que podem ser baixados diretamente do Google Play, Apple Store ou então pelo próprio site do aplicativo.

Este artigo apresenta o software Scratch (2020) que começou a ser usado recentemente no Brasil. O Scratch traz noções básicas de programação dentro do próprio aplicativo e nos possibilita fazer uma abordagem diferente sobre alguns conceitos matemáticos como plano cartesiano, ângulos, geometria analítica e geometria plana.

\section{MÉTODO}

Segundo Oliveira e Lima (2017), “a educação passa por uma profunda mudança sociocultural, já que ela é fruto da sociedade. Logo, se esta muda, aquela também mudará. Uma das grandes responsáveis por essa mudança é a tecnologia. Assim sendo, o ensino tradicional já não dá conta da sociedade tecnológica que vem se moldando ao longo dos anos. Muitos dos problemas educacionais vivenciados são fruto do desacerto existente entre metodologias antigas, ainda muito utilizadas em sala de aula, e a existência de alunos nativos digitais, que não veem mais o professor como o único detentor do conhecimento". O ensino de Matemática e mais especificamente, o de Geometria não fica fora dessa problemática. Diante dessa situação, é necessário que os professores busquem soluções de tal modo que o ensino da geometria seja eficiente em sua didática e divertida.

O Scratch é um projeto do grupo Lifelonge Kindergarten no Media Lab do MIT (Instituto de Tecnologia de Massachusets), onde foi idealizado por Mitchel Resnick. É 
um software que se utiliza de blocos lógicos, itens de som e imagem, para o usuário desenvolver suas próprias histórias interativas, jogos e animações, além de compartilhar de maneira online suas criações. (Scratch Brasil).

Ele foi projetado especialmente para idades entre 8 e 16 anos, mas é usado por pessoas de todas as idades, está sendo usado em mais de 150 países, disponível em mais de 40 idiomas, e é fornecido gratuitamente para os principais sistemas operacionais (Windowns, Linux e Mac).

O uso do Scratch possibilita ao usuário manusear alguns conceitos de programação como sequência, iteração, condição, variáveis, execução paralela, sincronia, iteração em tempo real, lógica booleana, números randômicos, tratamento de evento e criação de interface.

Figura 1. Interface do software Scratch com script de dois objetos e cenário.

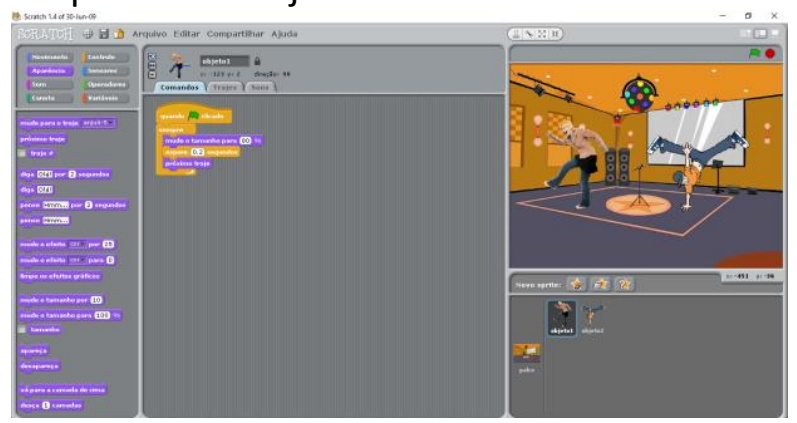

Além programação retratados acima, o aplicativo possibilita por meio dos comandos movimento, aparência, som, caneta, controle, sensores, operadores e variáveis os recursos essenciais para realizar operações matemáticas, construções de figuras geométricas, alterar as coordenadas cartesianas, raciocínio lógico através do comando "se, senão" e movimentos dos objetos. Pode-se desenvolver a criatividade dentro do software, como por exemplo, criações de programas que sistematizam simultaneamente animações, músicas, manejo de mídias, sons e gráficos. $E$, apresenta um importante recurso de compartilhamento de suas ações dentro do próprio site online, favorecendo assim o caráter empreendedor dos jovens, bem como fomentar a socialização do conhecimento adquirido.

Figura 2. Coordenadas não encaixadas, apenas soltas na área de programação.

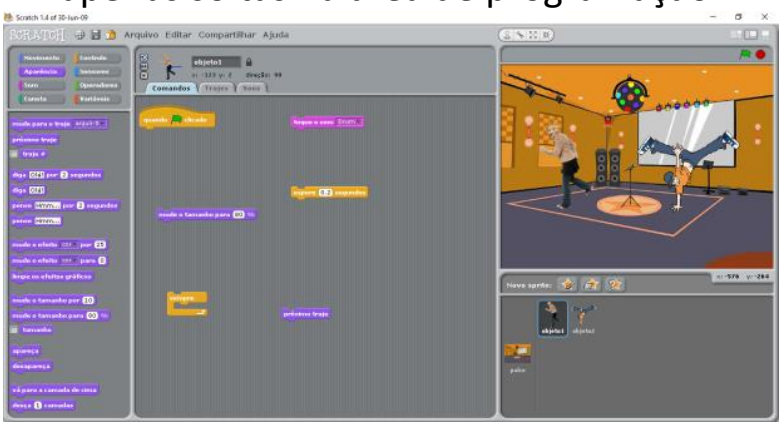

Figura 3. Coordenadas encaixados na área de programação formando "pilhas ordenadas".

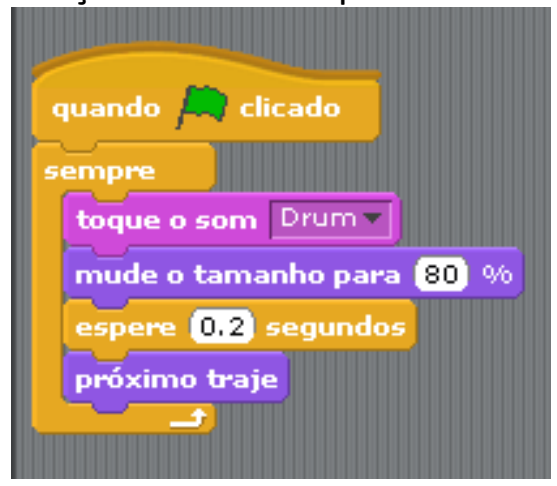

Uma das particularidades desta ferramenta é a capacidade de não realizar erros de construção, visto que não é fundamental teclar o comando, uma vez que o próprio vem em molde de segmentos em que basta deslizá-los. Da mesma forma, os segmentos são desenvolvidos para serem embutidos, assim, não ocorrendo junturas com erros. As sequências e as instruções podem ser alteradas mesmo com o programa em movimento, possibilitando assim a geração de uma nova ideia.

\section{RESULTADOS E DISCUSSÕES}

Neste tópico será realizado algumas atividades utilizando ao software Scratch para mostrar que o aplicativo pode ser utilizado nas séries do ensino fundamental, com o objetivo de implementar uma didática diferente do que a tradicional forma de ensinar que estamos habituados. 
Primeiramente, seguindo as orientações do professor, os alunos criarão uma conta no Scratch. Em seguida, entrarão na classe criada pelo professor, e finalmente, realizarão as atividades proposta pelo profissional.

3.1. Construção de um quadrado cujo perímetro é de 400 passos (unidade de medida do software)

A série a ser trabalhada será o $7^{\circ}$ ano do Ensino Fundamental. O tempo estimado para a realização da atividade será de 25 minutos, será necessário à utilização da sala de tecnologia e o uso de um retroprojetor. 0 tema a ser trabalhado será Cálculo de Perímetro, desta forma, construir-se-á um quadrado utilizando o aplicativo com o objetivo de fazer com que o aluno coloque em prática as noções de perímetro.

Figura 4. Coordenadas do quadrado.

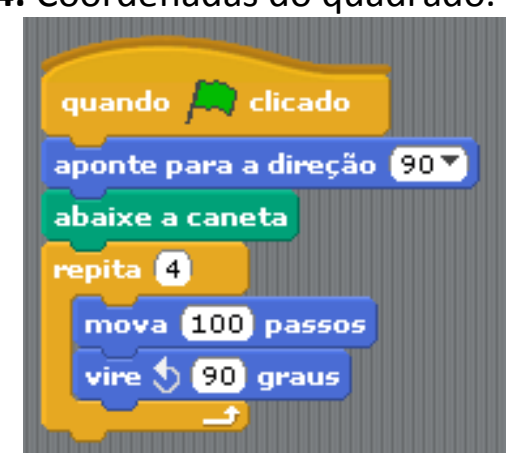

Após realizar esses passos, observa-se que o cenário ficou parecido com da figura 5 .

Figura 5. Quadrado com 400 passos de perímetro.

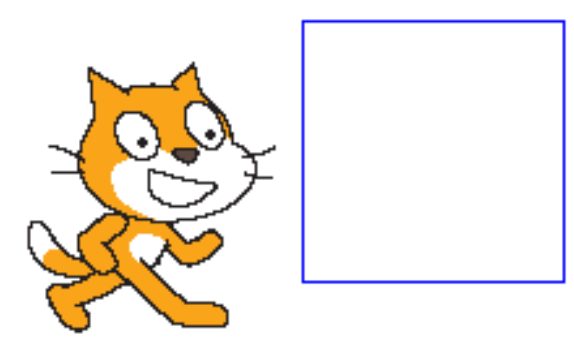

Notemos que a caneta andou 100 passos e logo em seguida virou 90 graus à esquerda, repetindo a mesma ação quatro vezes até formar o quadrado. Observe que

$$
100+100+100+100=400
$$

assim, espera-se que 0 aluno realize as noções de perímetro somando as medidas dos lados do quadrado.

\subsection{Construção de um retângulo cuja área é de $\mathbf{2 0 . 0 0 0}$ passos.}

A série a ser trabalhada será o $7^{\circ}$ ano do Ensino Fundamental. O tempo estimado para a realização da atividade será de 25 minutos, será necessário à utilização da sala de tecnologia e o uso de um retroprojetor. 0 tema a ser trabalhado será o Cálculo de Área por intermédio do Scratch. Nesta atividade espera-se que os alunos saibam os conceitos de como calcular a área de um retângulo, pois o objetivo da atividade é que o aluno programe a partir do que lhe foi apresentado.

Sabe-se que existem várias
possibilidades de encontrarmos um
retângulo cuja área é de 20.000 passos, uma
delas é utilizando os comandos como mostra
a figura 6 .

Figura 6. Coordenadas do retângulo.

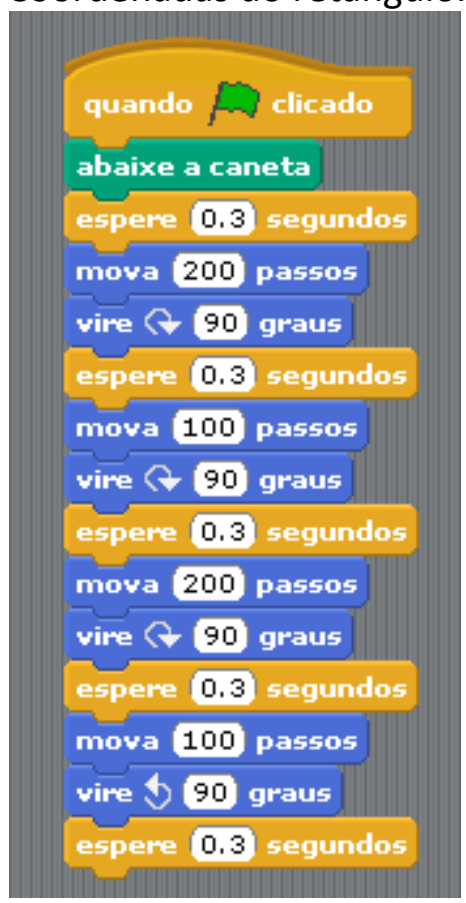

Após realizar esses passos, observe que o cenário ficou parecido com da figura 7. 
Figura 7. Retângulo

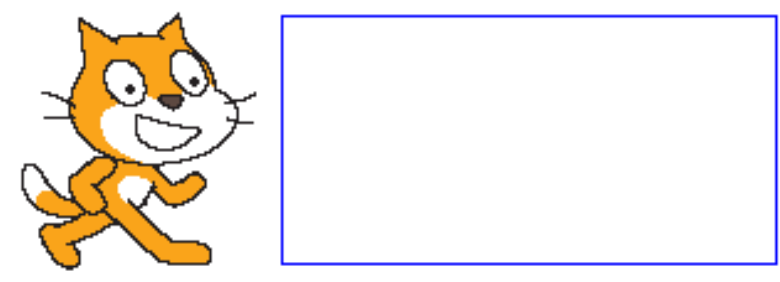

\subsection{Construção do ângulo de $135^{\circ}$.}

A série a ser trabalhada será o $7^{\circ}$ ano do Ensino Fundamental. O tempo estimado para a realização da atividade será de 25 minutos, será necessário à utilização da sala de tecnologia e o uso de um retroprojetor. 0 tema a ser trabalhado será Construção de ângulos, desta forma, construir-se-á ângulos utilizando nosso aplicativo com o objetivo de fazer o aluno identificar a abertura do ângulo como grandeza relacionada às figuras geométricas, solucionar problemas que envolvam a noção de ângulo e estabelecer medidas de ângulos; aplicar as ideias de ângulos suplementares e adjacentes.

Irão construir o ângulo de $135^{\circ}$ utilizando a seguinte programação:

Figura 8. Coordenadas do ângulo de $135^{\circ}$.

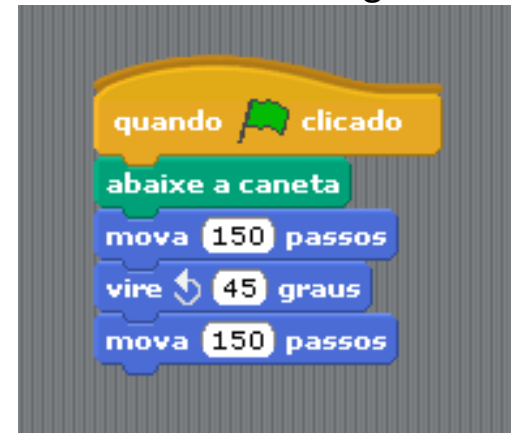

Após realizar estes passos, observe que o cenário é parecido com o da figura 9.

Figura 9. Ângulo de $135^{\circ}$.
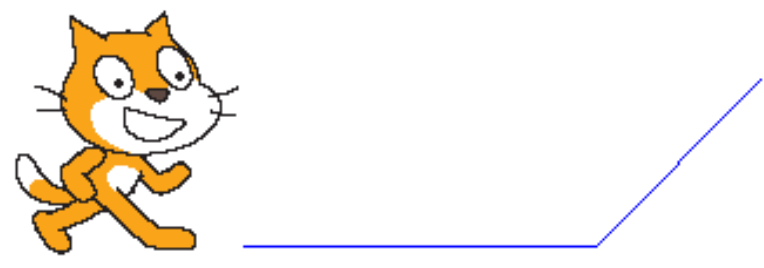

Observe que a caneta andou 100 passos e logo em seguido virou $45^{\circ}$ à esquerda e andou mais 100 passos, desta forma, espera-se que o aluno realize as noções de ângulos suplementares, uma vez que $180^{\circ}-45^{\circ}=135^{\circ}$.

\subsection{Construção de ângulos especiais}

A série a ser trabalhada será o $7^{\circ}$ ano do Ensino Fundamental. O tempo estimado para a realização da atividade será de 25 minutos, será necessário a utilização da sala de tecnologia e o uso de um projetor. O tema a ser trabalhado será Construção de ângulos por intermédio do Scratch. Nesta atividade espera-se que os alunos saibam os conceitos de ângulo reto, agudo, obtuso, suplementar e complementar, pois o objetivo da atividade é que o aluno programe a partir do que lhe foi apresentado, de modo que seu personagem represente ângulos com os atributos pedidos.

Com este exercício, o professor será capaz de analisar se ocorreu um entendimento, por parte do aluno, das concepções matemáticas e de programações apresentadas.

\subsection{Construção de qualquer polígono equilátero.}

A série a ser trabalhada será o $7^{\circ}$ ano do Ensino Fundamental. O tempo estimado para a realização da atividade será de 25 minutos, será necessário à utilização da sala de tecnologia e o uso de um retroprojetor. 0 tema a ser trabalhado será Construção de qualquer construção de um polígono equilátero. Nesta atividade espera-se que o aluno reconheça os ângulos do polígono e efetue os movimentos corretos para construí-los, utilizando a soma dos ângulos internos de um triângulo, de um pentágono e de um hexágono, e determine uma relação entre as medidas do lado.

Existem várias possibilidades para a construção dos polígonos equiláteros, o conceito da atividade é que o aluno produza táticas para tentar fazer o polígono, assim, desenvolver habilidades e conhecimentos em 
Geometria de forma dedutível. Um possível resultado está nas figuras a seguir.

Figura 10. Coordenadas do triângulo.

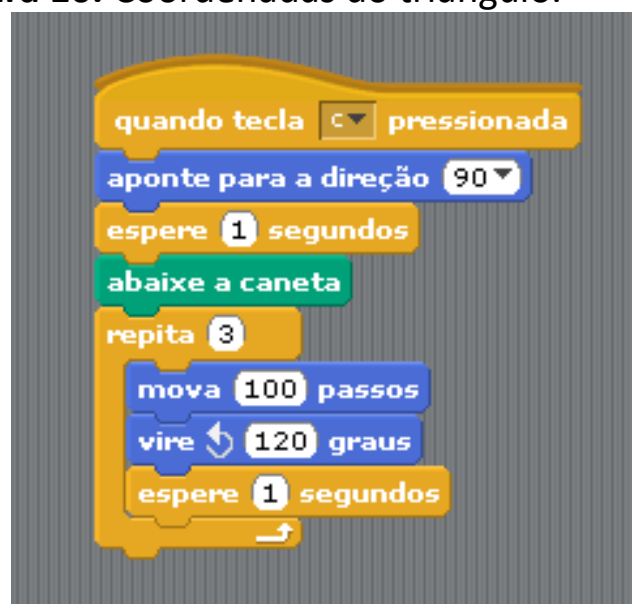

Figura 11. Triângulo equilátero.
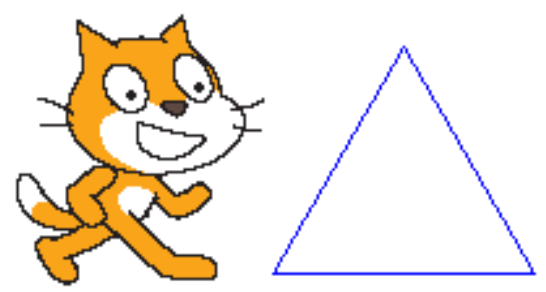

Figura 12. Coordenadas do pentágono

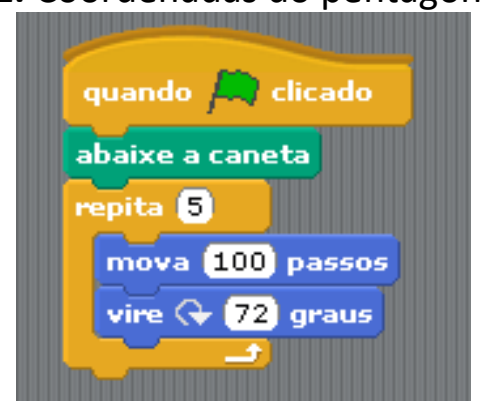

Figura 13. Pentágono equilátero.

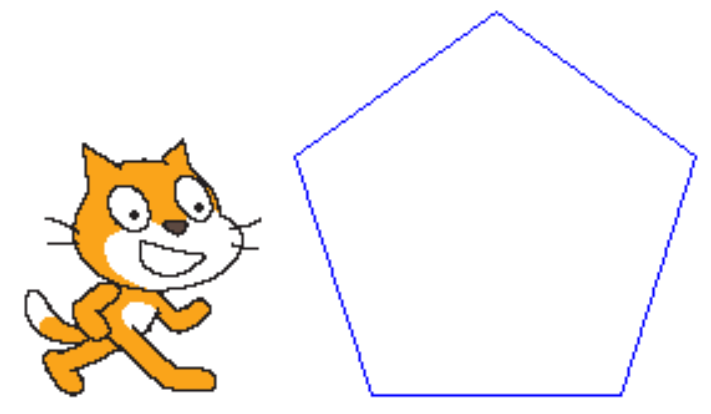

Figura 14. Coordenadas do hexágono

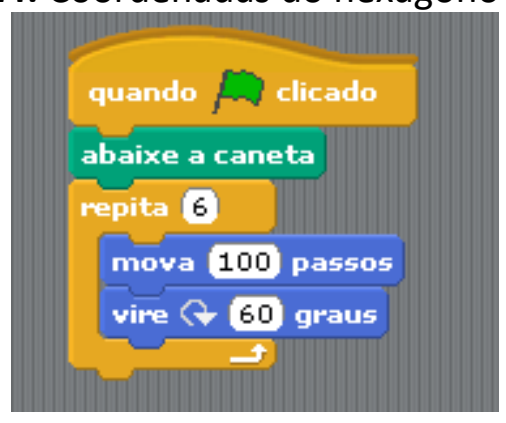

Figura 15. Hexágono equilátero.

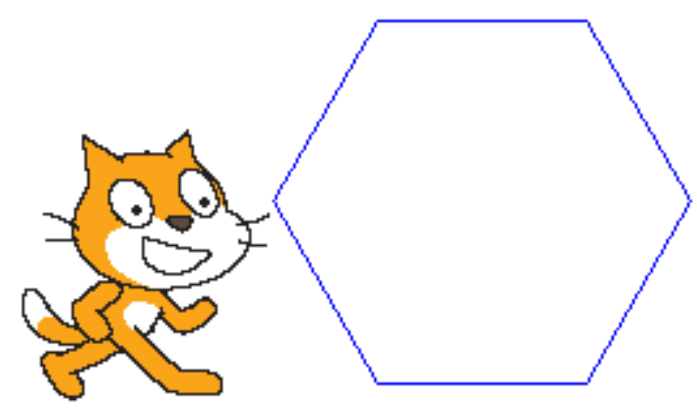

\section{CONSIDERAÇÕES FINAIS}

Através do presente artigo foi possível comentar sobre a importância do uso da tecnologia na educação brasileira, apresentar o Software Scratch e mostrar que o professor de matemática do ensino básico possui alternativas para ensinar a Geometria de uma maneira mais dinâmica utilizando o Software Scratch, tal como construção de ângulos e polígono, cálculo de áreas e perímetro utilizando a linguagem computacional do aplicativo.

\section{AGRADECIMENTOS}

Programa de Educação Tutorial, Universidade Federal de Mato Grosso do Sul.

\section{REFERÊNCIAS}

CÂMARA DOS DEPUTADOS. Projeto de Lei $\mathbf{n}^{\circ}$ 9.165, de 2017. Brasília, DF: Câmara dos Deputados, 2017. Disponível em: https://www.camara.leg.br/proposicoesWeb/pro p mostrarintegra; jsessionid=AC4D3E73DECCF990 455F75C5354A4E89.proposicoesWebExterno2?C odteor $=1675485 \&$ filename $=$ Avulso+PL+9165/2017 . Acesso em: 17 jan. 2020. 
MARQUES, J. C. O. Construção de mosaicos utilizando a linguagem de programação Scratch como ferramenta para o ensino de Geometria Plana. 2019. Dissertação (Mestrado Profissional em Matemática em Rede Nacional) - PROFMAT da Universidade Tecnológica Federal do Paraná, Cornélio Procópio, PR, 2019.

OLIVEIRA, H. S.; LIMA, M. F. W. P. Utilização da Plataforma Khan Academy na Resolução de Exercícios de Matemática., Scientia Cum Industria, v. 5, n. 2, p 66-72, 2017. https://doi.org/10.18226/23185279.v5iss2p6 $\underline{6}$

SCRATCH BRASIL. Scratch Brasil. Scratchbrasil.net.br. Disponível em: index.php/sobre-o-scratch.html. Acesso 13 jan. 2020.

TUDOCELULAR.COM. Pesquisa revela que 97\% dos estudantes utilizam smartphones para acessar a internet. Tudo Celular.com. 22 ago. 2018. Disponível em: https://www.tudocelular.com/tech/noticias /n129645/estudo-alunos-conectadosescolas-wifi.html. Acesso em: 15 jan.2020.

VARELLA, G. Há laboratórios de informática em $81 \%$ das escolas públicas, mas somente 51\% são usados. Época, 3 ago. 2017. Disponível em: https://epoca.globo.com/educacao/noticia/2 017/08/ha-laboratorios-de-informatica-em81-das-escolas-publicas-mas-somente-59sao-usados.html. Acesso em: 15 jan. 2020.

VENTORINI, A. E./; FIOREZI, L. A. Software Scratch: uma contribuição para o ensino e aprendizagem da Matemática. In: EIEMAT ESCOLA DE INVERNO DE EDUCAÇÃO MATEMÁtICA. 4., 2014, Santa Maria, RS. Anais [...]. Santa Maria: UFSM 2014. 